\title{
Mejora del Control de un IPMSM con Tensión de Batería Baja
}

\author{
Alberto Sanz, Estanis Oyarbide, David Owtschinnikow \\ Group of Power Electronics and Microelectronics (GEPM) \\ Instituto de Investigación en Ingeniería de Aragón (I3A) \\ Universidad de Zaragoza, Mariano Esquillor s/n, 50018, Zaragoza, Spain. \\ Tel. +34-976762707, e-mail: 473556@,celes.unizar.es
}

\begin{abstract}
En este documento se presenta el control vectorial de un motor síncrono de imanes permanentes insertados (IPMSM) utilizado en un sistema de dirección asistida eléctrica (EPS). El objetivo es optimizar el bloque que, según los requerimientos de par, genera las corrientes de referencia del motor.
\end{abstract}

\section{Introducción}

Los IPMSM son ampliamente utilizados en sistemas de a bordo debido a su alto rendimiento en un diseño compacto y robusto. En este trabajo, el IPMSM forma parte de una EPS (Fig.1) para coches pequeños. La estrategia de control implementada establece rápidamente las condiciones de operación que permiten que el motor proporcione el par requerido por el usuario. Esto se hace a través de un lazo interno de control de corriente y un bloque de cálculo de corriente de referencia, (Fig. 2).

Uno de los problemas con el que una EPS debe lidiar es el sobrecalentamiento del motor y las placas electrónicas, que depende de las corrientes del motor. Por esta razón los sistemas EPS necesitan determinar la amplitud y posición de la mínima corriente que proporcione el par requerido, aprovechando la llamada estrategia de Máximo Par Por Amperio (MTPA). La mayor dificultad aparece a altas velocidades, donde se requieren estrategias de debilitamiento de campo debido a la saturación de la tensión, implicando el uso de la estrategia de Máximo Par Por Voltio (MTPV).

En este motor, al ser de imanes insertados, es posible aumentar la capacidad de par de la EPS aprovechando el "par reluctante" $(\mathrm{Ld} \neq \mathrm{Lq})$. Sin embargo, esto complica las relaciones analíticas del motor. Por otra parte, el rango de tensión de la batería es de $7 \mathrm{~V}$ a $18 \mathrm{~V}$, mientras que las corrientes del motor son altas (100A). Así, a diferencia de motores operando a niveles de tensión industrial, las resistencias de los devanados deben ser consideradas en las ecuaciones de tensión. Estos problemas han sido estudiados previamente con diferentes enfoques. La mayoría descuida la resistencia de los devanados y considera imanes de montaje superficial $(\mathrm{Ld}=\mathrm{Lq})$, [1] y [2]. [3] Tiene en cuenta las resistencias de los devanados y el par reluctante, pero no opera en la región MTPV, por lo que el enfoque no cubre la región más exigente en escenarios a baja tensión.

En este trabajo, el desarrollo del bloque de generación de la corriente de referencia se llevó a cabo teniendo en cuenta el par reluctante y las resistencias de fase. La tensión de batería ha sido considerada como una tercera variable de entrada, junto con la velocidad del motor y el par de referencia. Se calcula la corriente de referencia a través de un algoritmo basado en las expresiones analíticas del motor exactas.

\section{Estrategia propuesta}

El par entregado por el motor de imanes permanentes viene dado por la ecuación (1),

$$
\Gamma\left(i_{d}, i_{q}\right)=\frac{3}{2} p i_{q}\left[\varphi+\left(L_{d}-L_{q}\right) i_{d}\right]
$$

donde $\mathrm{p}$ es el número de pares de polos, $\phi$ es el flujo de los imanes permanentes, Ld y Lq son las inductancias directa y de cuadratura respectivamente y id y iq son las corrientes directa y de cuadratura, respectivamente. El objetivo del algoritmo es identificar la corriente más apropiada. Dependiendo del modo de operación de la máquina (temperatura y velocidad) y del nivel de tensión de la batería, la corriente máxima disponible variará, por lo que el par requerido será alcanzable o no. Si el par de referencia es alcanzable, la corriente más adecuada será la mínima que asegure el par requerido, por lo tanto, la corriente con el mínimo impacto térmico. Por otro lado, si el par requerido no es alcanzable, la corriente más adecuada será la que implique el máximo par posible, minimizando así, el error de par.

\section{Resultados de simulación}

El algoritmo anteriormente descrito se implementó en $\mathrm{C}$ utilizando la herramienta S-Function de Matlab-Simulink. La Fig. 3 muestra la gráfica par 
resultante-velocidad bajo cuatro tensiones de batería diferentes, mostrando la flexibilidad del algoritmo. El tiempo de ejecución del algoritmo ha sido medido usando dos plataformas diferentes: a) dSPACE 1103, cuenta con un Power PC 750 GX a $1 \mathrm{GHz}, \mathrm{y}$ b) un PC dual core a $2.933 \mathrm{GHz}$. En el caso a) el algoritmo require 6.93 $\mu \mathrm{s}$ (6930 ciclos de reloj), mientras que en el caso b) el tiempo de ejecución baja a $2.71 \mu s$ (7939.3 ciclos de reloj).

\section{Conclusiones y trabajo en curso}

El algoritmo desarrollado proporciona la corriente de referencia mínima requerida para cada par de referencia, reduciendo así el calentamiento del motor. Contrariamente a otros trabajos publicados, este trabajo tiene en cuenta todas las variables implicadas y todos los parámetros del motor. Teniendo en cuenta que un muestreo y un período de control de $100 \mu$ s son suficientes en el control de este tipo de máquinas, el método propuesto de generación de corriente es una solución prometedora para el control de unidades IPMSM en sistemas de a bordo, por lo que, se ha implementado el algoritmo en una dSPACE a través de Matlab-

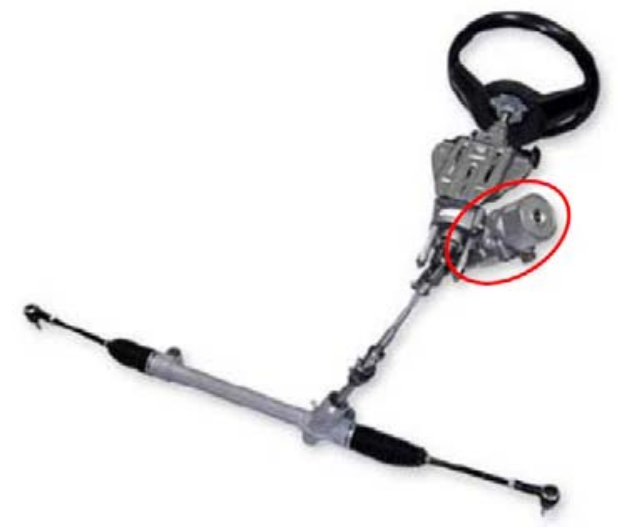

Fig.1. EPS tipo columna con IPMSM.

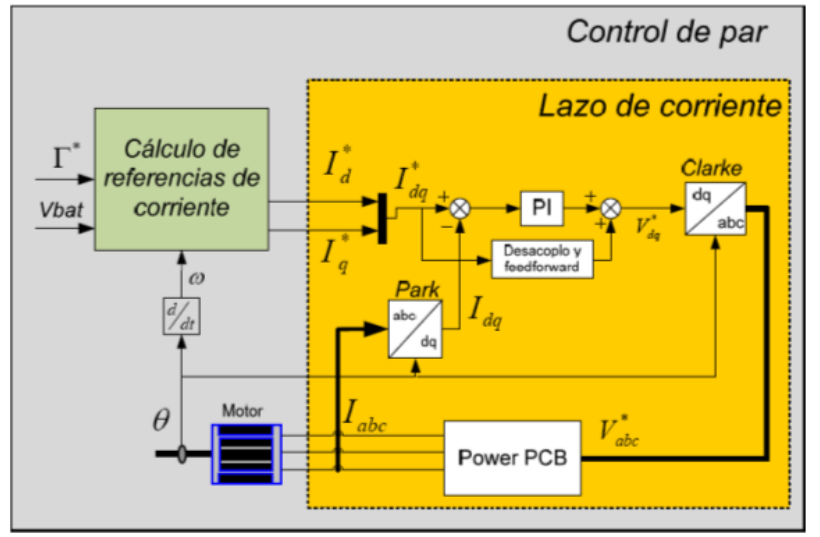

Fig.2. Esquema del bloque de control de par.
Simulink, controlando así un IPMSM, con el objetivo de realizar las pruebas necesarias para poder validar los resultados de las simulaciones. La bancada de pruebas utilizada se muestra en la Fig. 4.

\section{REFERENCIAS}

[1]. SHUGEO MORIMOTO, YOJI TAKEDA, TAKAO HIRASA, KATSUNORI TANIGUCHI; "Expansion of Operating Limits for Permanent Magnet Motor by Current Vector Control Considering Inverter Capacity"; IEEE Transactions on Industry Applications, Vol.26, No.5, September-October 1990, pp.866-871.

[2]. THOMAS M. JAHNS, "Flux-Weakening Regime Operation of an Interior Permanent-Magnet Synchronous Motor Drive"; IEEE Transactions on Industry Applications, VOL. IA-23, NO. 4, JULY/AUGUST 1987, pp. 681-689.

[3]. CHING-TSAI PAN, SHINN-MING SUE; “A Linear Maximum Torque Per Ampere Control for IPMSM Drives Over Full-Speed Range"; IEEE Transactions on energy conversion, Vol.20, No.2, June 2005, pp.359-366.

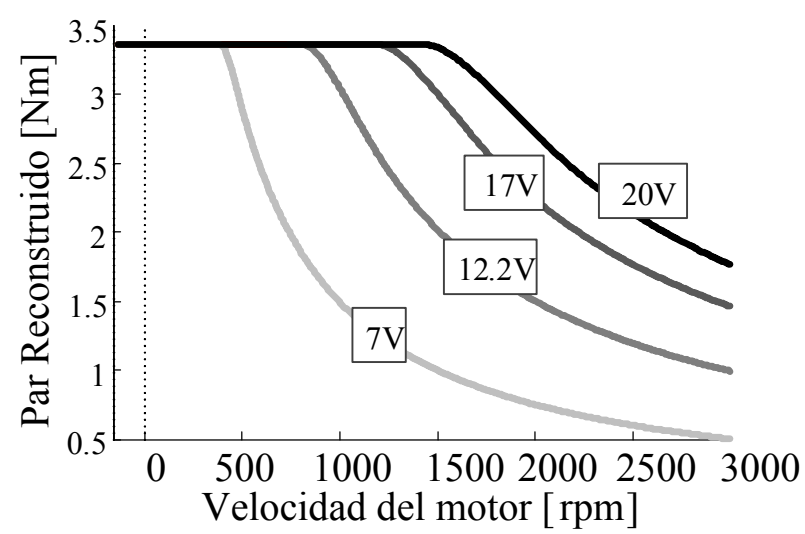

Fig. 3. Gráficas par resultante-velocidad.

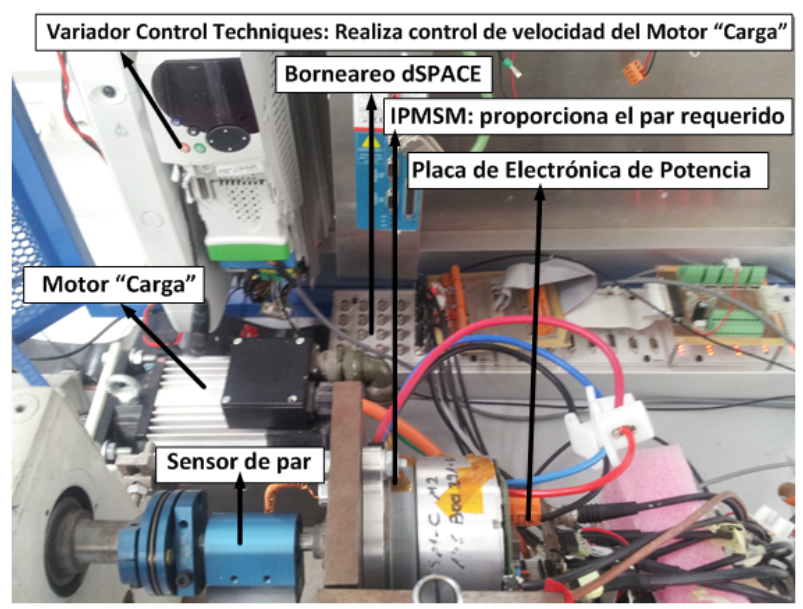

Fig. 4. Bancada de pruebas. 